\title{
DESENVOLVIMENTO, CONSTRUÇÃO E CALIBRAÇÃO DE UMA CENTRAL DE MONITORAMENTO DE CONSUMO DE ENERGIA ELÉTRICA E DE ÁGUA UTILIZANDO O MICROCONTROLADOR ARDUÍNO.
}

\author{
M.C.Ramos ${ }^{1 *}$; V.S.Andrade ${ }^{1}$
}

1 Instituto Federal de Minas Gerais, 30575-180, Governador Valadares-MG, Brasil

*mateuscontim@gmail.com

Artigo submetido em 23/09/2015 e aceito em 03/03/2016

\begin{abstract}
RESUMO
O presente trabalho apresenta os resultados do desenvolvimento de instrumentação que possibilita a medição suficientemente precisa dos consumos de energia elétrica e de água de uma instalação de pequeno porte. Os protótipos propostos permitem a medida da potência elétrica consumida da rede e da vazão de água drenada do sistema de abastecimento como funções do tempo. Através das curvas temporais de potência e vazão é possível a obtenção, via cálculo numérico, do consumo de energia e de água. Dado o problema social gerado pela crise energética e hídrica, um dos objetivos do trabalho é atender uma carência de medidas físicas que permitam, com baixo custo, o melhor gerenciamento de ações de
\end{abstract}

conservação de energia e água. Em razão do contorno de baixo custo e opção por um sistema open source, foi utilizado o microcontrolador Arduíno. Além disso, objetiva-se a utilização futura do instrumental e os respectivos conhecimentos adquiridos, como objeto de ensino no curso de engenharia de produção. São apresentadas no corpo do trabalho as etapas de desenvolvimento, detalhes da construção e métodos de calibração de ambos os sensores. Os resultados obtidos no decorrer da calibração dos sensores indicam uma precisão compatível com as necessidades de medição e objetivos propostos.

PALAVRAS-CHAVE: Conservação de Energia, Conservação de Água, Instrumentação.

\section{DEVELOPMENT, CONSTRUCTION AND CALIBRATION A CENTRAL MONITORING ENERGY CONSUMPTION AND WATER USING ARDUINO MICROCONTROLLER}

\begin{abstract}
This paper presents the results of the development of instrumentation that enables sufficiently precise measurement of energy and water consumption of a small installation. The proposed prototypes allow the measurement of the electrical power consumed from the grid and the water flow drained from the supply system as functions of time. Through the temporal power and flow curves is possible to obtain, throughout numerical calculation (integration), the consumption of energy and water. Given the social problem created by the energy and water crisis, one of the objectives of the work is to find a shortage of physical measures that permit, at low
\end{abstract}

cost, better management of energy and water conservation actions. Because of the low cost contour and choice of an open source system, we used the Arduino microcontroller. In addition, the objective is the future use of the instruments and their acquired knowledge, as a teaching object in the course of production engineering. In the body are presented: the stages of development, construction details and both sensors calibration methods. The results obtained during the calibration of the sensors indicate an accuracy that is consistent with the measurement needs and proposed objectives.

KEYWORDS: Energy Conservation, Water Conservation, Instrumentation. 


\section{INTRODUÇÃO}

Ao longo da última década, o Brasil experimentou um constante crescimento econômico. Fundamentalmente a geração de energia é uma das premissas para alavancar o desenvolvimento social e econômico de um país. Com a ascensão das Classes D e C e a ampliação do parque fabril nos últimos anos, foi gerada uma elevação significativa do consumo de energia elétrica (ANEEL, 2014). É de conhecimento geral que o Brasil é um país com possibilidades de geração de energia a partir de um conjunto de fontes renováveis, visto que $80 \%$ do processo de sua obtenção energética provêm da hidroeletricidade, não obstante mesmo sendo a forma mais econômica de gerar energia, acaba por gerar impactos de natureza socioambiental em razão da construção de barragens de grande porte e remanejamento da população que se encontra próxima das áreas do empreendimento hidrelétrico (SILVA e MORET, 2012). Acrescente-se a isso o fato de o aproveitamento do notório potencial natural do país ser refém dos índices pluviométricos que ajudam a manter os níveis de reservatórios e a vazão dos rios.

Atualmente é possível perceber que a matriz energética nacional está defasada e debilitada pelas fortes estiagens dos últimos anos, o que gerou grande escassez de água nos reservatórios das hidrelétricas, comprometendo a chamada energia firme do sistema. Isso é em parte reflexo da forma de organização, produção e distribuição da energia elétrica, que não levou em consideração, pelo menos com a atenção que deveria, políticas de diversificação da matriz energética, planejamento energético e eficiência energética. As consequências têm atingido todo o arranjo produtivo nacional e a população com elevações severas e constantes na sua fatura mensal de energia. Para agravar ainda mais a situação, a queda dos índices pluviométricos mínimos gera outra crise de fornecimento, a de abastecimento de água. Esta crise hídrica, por sua vez, está fortemente correlacionada à baixa eficiência dos sistemas de abastecimento. Estima-se que no Brasil $37 \%$ da água tratada se perde no processo de fornecimento, enquanto nos países desenvolvidos temos perdas de $5 \%$ a $15 \%$. Tornou-se rotineiro o racionamento de água em inúmeras cidades brasileiras e em alguns casos há falta da mesma por períodos longos, proporcionando grandes transtornos para a população (TRATABRASIL, 2013).

Algumas consequências imediatas da crise energética e hídrica vigentes são o aumento de tarifas (consumidores residenciais e grandes empresas), uso constante de usinas térmicas (impacto ambiental e econômico severo) e maior custo nas tarifas de água para todos os consumidores. Essa questão afeta diretamente os custos de produção das empresas, pois todas elas possuem como insumos básicos a energia e a água. De acordo com (BERMANN, 2011), a análise do perfil industrial eletrointensivo do país indica que $30 \%$ da energia elétrica produzida no Brasil é consumida por apenas seis ramos industriais: cimento, ferro-gusa e aço (siderurgia), ferro ligas, não ferrosos (alumínio), química, papel e celulose. Sendo setores produtivos que se caracterizam pela produção de produtos destinados à exportação, gerando divisas para o país. Torna-se indispensável para a transformação do país a formação de projetos energéticos e hídricos eficientes, para que a população e as empresas tenham acesso a esses insumos básicos a um valor competitivo e com maior qualidade.

Neste contexto, o controle e o gerenciamento de perdas através de ações de conservação de energia e água se tornam indispensáveis para as empresas e para a população em geral. Segundo (KEELLING, 2012), o gerenciamento de perdas carece primariamente de medidas que sejam a base para o gestor analisar os gastos e propor alternativas (desligamento de equipamentos, substituição de equipamentos, alteração de processos produtivos, políticas de conscientização, entre outros). Tendo em vista essa questão, o presente trabalho procurou desenvolver um instrumento eletrônico de baixo custo que seja capaz de medir o consumo de energia elétrica e de água de forma 
simultânea, gerando dados (planilhas e gráficos de consumo em função do tempo) para o desenvolvimento de possíveis projetos de pesquisa e extensão envolvendo a temática citada. Além disso, o projeto visa o levantamento de dados sobre o consumo de energia e de água de uma instituição de ensino: IFMG - Câmpus Governador Valadares. Neste caso, pretende-se auxiliar as atividades da CICE - Comissão Interna de Conservação de Energia.

As atividades de desenvolvimento do projeto ocorreram em dois ambientes: a residência de um dos autores e o Laboratório de Ciências Naturais da instituição de ensino, onde os respectivos testes e ajustes foram feitos. Pensando nas dificuldades que muitas vezes surge no ensino de engenharia e na busca por uma interdisciplinaridade, uma expectativa do projeto era empregar boa parte dos conhecimentos adquiridos no curso de Engenharia de Produção (Eletrônica, Cálculo Numérico, Programação, Estatística, Física) na produção de protótipos que fossem capazes de auxiliar num problema de gestão bastante atual: conservação de energia (Eficiência Energética) e água (Eficiência Hídrica). Tudo isso numa visão integradora e como passo inicial de ações nesse sentido. Além disso, foi escolhida uma forma de baixo custo, de código aberto (Open Source), o microcontrolador Arduíno, que se caracteriza por sua flexibilidade, acessibilidade e pela perspectiva de uso futuro dos protótipos gerados como objetos de ensino. É preciso despertar o interesse dos alunos do curso de Engenharia de Produção e de outros cursos da instituição pelo estudo dessa temática, levando o conhecimento adquirido no decorrer do desenvolvimento do projeto a toda a comunidade acadêmica do IFMG - Câmpus Governador Valadares através de palestras e oficinas (destinadas aos alunos interessados). A plataforma arduíno é composta de hardware e software livres, sendo assim, possui uma rede de cooperativismo e solidariedade entre os usuários com livre compartilhamento de informações, esquemas lógicos, compartilhamento de resultados e suporte para a solução de problemas e/ou dúvidas, através de fóruns, sites e redes sociais, democratizando, assim, o conhecimento, como preconiza o movimento Open Source (DA SILVEIRA, 2012).

Logo o presente trabalho possui o objetivo central de desenvolver um aparato de instrumentação de baixo custo para o uso no controle de consumo de energia e água de uma instalação. Além disso, auxiliará o ensino de engenharia através da construção de um protótipo como objeto de ensino, que poderá ser utilizado para replicação em oficinas (Eletrônica, Física, Automação) e geração de dados que sejam subsídios para conservação de água e energia (CICE). Outros objetivos referentes ao projeto são a aquisição e compartilhamento dos conhecimentos acerca do microcontrolador Arduíno e de sua linguagem de programação, fazendo uso de uma plataforma open souce e ambiente colaborativo, servindo como pilar para a elaboração de novos projetos referentes nessa linha, e aplicação dos conhecimentos adquiridos no decorrer do curso de graduação em Engenharia de Produção (Programação de Computadores, Administração, Engenharia do Produto, Cálculo Numérico, Metrologia, Eletrônica e Física) para solução de um problema de engenharia numa perspectiva interdisciplinar.

\section{MATERIAIS E MÉTODOS}

Dado o contorno de baixo custo, o objetivo central de criação de um sistema que auxilie na conservação de energia elétrica e água, e a questão da construção de um sistema que envolva os alunos através de um ensino por projetos dentro de um ambiente multidisciplinar e colaborativo, o microcontrolador e seus respectivos elementos sensores foram escolhidos com base nesses critérios. Foi realizado um processo de pesquisa para subsidiar a escolha dos componentes necessários para a construção dos protótipos, e foi possível concluir que o microcontrolador Arduíno supera seus concorrentes diretos (Raspberry Pi e Intel Galileo), por ser um hardware livre e com código fonte aberto, proporcionando uma facilidade de encontrar sensores que devidamente programados supram as necessidades do usuário com poucas alterações e/ou adaptações na programação. Além disso, o 
fator econômico foi fundamental, pois como já dito, o intuito é de desenvolver um protótipo de baixo custo e acessível para reprodução e uso futuro como objeto de ensino.

Tecnicamente, a placa Arduíno pode ser definida como um pequeno computador que se pode programar para processar entradas e saídas entre o dispositivo e os componentes externos conectados a ele. Os componentes de entrada podem ser sensores, botões, potenciômetros, que podem ser conectados a entradas analógicas e/ou digitais. Os componentes de saída podem ser displays, LED's, motores, cartões de memória entre outros componentes (MCROBERTS, 2011). A placa utilizada no projeto foi a Arduíno UNO R3.

Para que se possa programar o Arduíno, é necessária a utilização do seu IDE (Integrated Development Environment), ou seja, um ambiente integrado para desenvolvimento de software. O mesmo é responsável por receber o código na linguagem que o microcontrolador compreende (baseado na linguagem $\mathrm{C} / \mathrm{C}++$ ) e transmiti-la para que o Arduíno execute a ação programada. E ainda na IDE é que ocorre a rotina responsável por exibir ao usuário os dados que estão sendo coletados pelos sensores.

Dada a necessidade de compatibilidade com a placa Arduíno, o sensor escolhido para medida de vazão, e consequente cálculo do consumo de água, foi o modelo YF - 21, que possui uma bitola de $1 / 2$ polegada. $O$ sensor é constituído por um corpo de válvula de plástico, um rotor, e um sensor de Efeito Hall. Quando a água flui através do rotor, passando da entrada à saída do sensor, o fluxo de água faz com que o rotor gire provocando a geração de tensão no sensor de Efeito Hall. Tal tensão é gerada de forma pulsada em função do número de giros do rotor. Como o giro depende da vazão que passa pelo sensor, há emissão de sinal pulsado com período dependente da vazão. Importante lembrar que o sensor possui rosca nas duas extremidades podendo ser facilmente adaptado a tubulações de diâmetros diferentes, como 3/4 de polegada por exemplo. Conforme folha de dados, o sensor trabalha com a faixa de vazão entre 1 a 30 litros por minuto. Para exibição da vazão a placa Arduíno "realiza a contagem dos pulsos gerados em determinado valor de tempo" e via calibração associa o volume que passa por unidade de tempo, interpretando assim qual a vazão que está sendo medida. Através de outros cálculos provenientes do código-fonte que foi desenvolvido, o Arduíno irá armazenar o volume acumulado em uma variável e irá exibi-lo a cada segundo. O consumo (volume) é encontrado através da integração da vazão volumétrica (quantidade de material transportado através de uma tubulação em litros) em função do tempo (segundos).

Para o cálculo da potência consumida foi escolhido um sensor de corrente não invasivo modelo SCT-013-000. Este modelo foi escolhido por ser compatível com a plataforma e devido a sua facilidade de ser clipado ao circuito de testes sem a necessidade de ligação entre fios, tal como um alicate amperímetro: abraçando o fio por onde passa a corrente. A premissa para essa modelagem é que a medida da corrente permite a obtenção da potência considerando que a tensão seja minimamente estável e desprezando-se os efeitos de fator de potência (carga em corrente alternada). Segundo o datasheet do sensor, o mesmo trabalha em uma escala de $50 \mathrm{~mA}$ a $100 \mathrm{~A}$ e temperaturas entre $-25^{\circ} \mathrm{C}$ e $70^{\circ} \mathrm{C}$. O sensor permite a leitura da corrente operando como um transformador de corrente, onde o primário é a rede elétrica. Segundo (TIPLER, 2006), para uma carga aproximadamente resistiva (fator de potência nas proximidades do unitário) a potência em corrente alternada pode ser encontrada pelo produto da tensão elétrica eficaz (volts) e a corrente elétrica eficaz (amperes) alimentam a carga. Já a energia consumida (Quilowatt-hora), é encontrada através da área do gráfico (integração) potência elétrica $(\mathrm{kW})$ em função do tempo $(\mathrm{h})$.

A programação para a leitura de dados dos dois sensores passou por duas grandes etapas: aquisição dos dados e armazenamento dos dados. No caso do sensor de vazão foi elaborada rotina para contagem de pulsos do sensor, que após calibração permitiu associar ao número de pulsos o 
volume que passa pelo sensor em uma dada unidade de tempo (vazão). Já no caso do sensor de potência foi preciso recorrer a uma rotina, disponibilizada por (OPENENERGYMONITOR, 2013), que permite obter o valor RMS do sinal de corrente alternada $(60 \mathrm{~Hz})$. Para o armazenamento dos dados em cartão de memória recorreu-se à instrução sobre esse procedimento disponível em (BRARDUINO, 2014). Importante lembrar aqui as grandes contribuições de desenvolvedores do Brasil e do mundo que esclareceram muitas dúvidas sobre algumas falhas encontradas.

Para a calibração do sensor de vazão foram necessárias inúmeras medições conforme o procedimento descrito sucintamente abaixo:

1 - O sensor é conectado ao Arduíno e encaixado em uma tubulação de 1/2 polegada. Foi utilizada fita veda rosca para eliminar possibilidades de vazamento.

2 - Uma torneira que está conectada ao ramal onde foi instalado o sensor foi aberta e a água era drenada para dentro de uma proveta graduada com intervalos de $100 \mathrm{~mL}$ e um volume máximo de 2 litros.

3 - O volume de água coletado na jarra foi obtido em um intervalo de tempo igual há um minuto, servindo de base de tempo para obtenção da vazão.

4 - Após várias repetições dos passos anteriores (total de 20), a vazão medida com o uso da proveta foi comparada com o número de pulsos registrados pela Arduíno.

5 - Um coeficiente para conversão do número de pulsos em vazão é obtido, permitindo que o resultado seja exibido em litros/s.

Para a calibração do sensor de potência elétrica foi feito o seguinte procedimento:

1 - O sensor é conectado ao Arduíno e o mesmo envolve (abraça) um único fio responsável pela alimentação de uma lâmpada incandescente com $60 \mathrm{~W}$ de potência utilizada como carga. Devido ao baixo valor de corrente optou-se por melhorar o fluxo magnético, e consequentemente a precisão na leitura, utilizando-se 6 voltas de fio sobre o sensor. Contudo, para evitar erro na leitura a corrente lida foi dividida por 6 no algoritmo para obtenção da potência instantânea. As leituras foram armazenadas.

2 - Em paralelo à medida do sensor, um simples amperímetro digital calibrado foi acoplado para medir a corrente do circuito com o propósito de verificar a precisão do sensor, para calcular o valor de potência da carga (lâmpada em teste), foi considerado uma tensão eficaz constante e igual a 127 V (segundo a equação de energia consumida).

3 - Utilizando o resultado obtido com o uso do amperímetro foi possível verificar que o sensor de potência estava fazendo uma leitura correta da grandeza (corrente elétrica).

\section{RESULTADOS E DISCUSSÃO}

\subsection{Algoritmo de Programação}

A programação dos sensores foi elaborada de modo que os dois sensores trabalhassem de forma integrada na mesma placa Arduíno. Além da programação para leitura, tratamento e acondicionamento dos sinais medidos, utilizou-se uma biblioteca de programação específica do Arduíno, chamada EmonLib.h, disponível para download em (OPENENERGYMONITOR, 2013), responsável pela leitura do sinais de tensão variantes no tempo $(60 \mathrm{~Hz})$. Na figura 1 está exposto o código de programação instalado na placa Arduíno. 


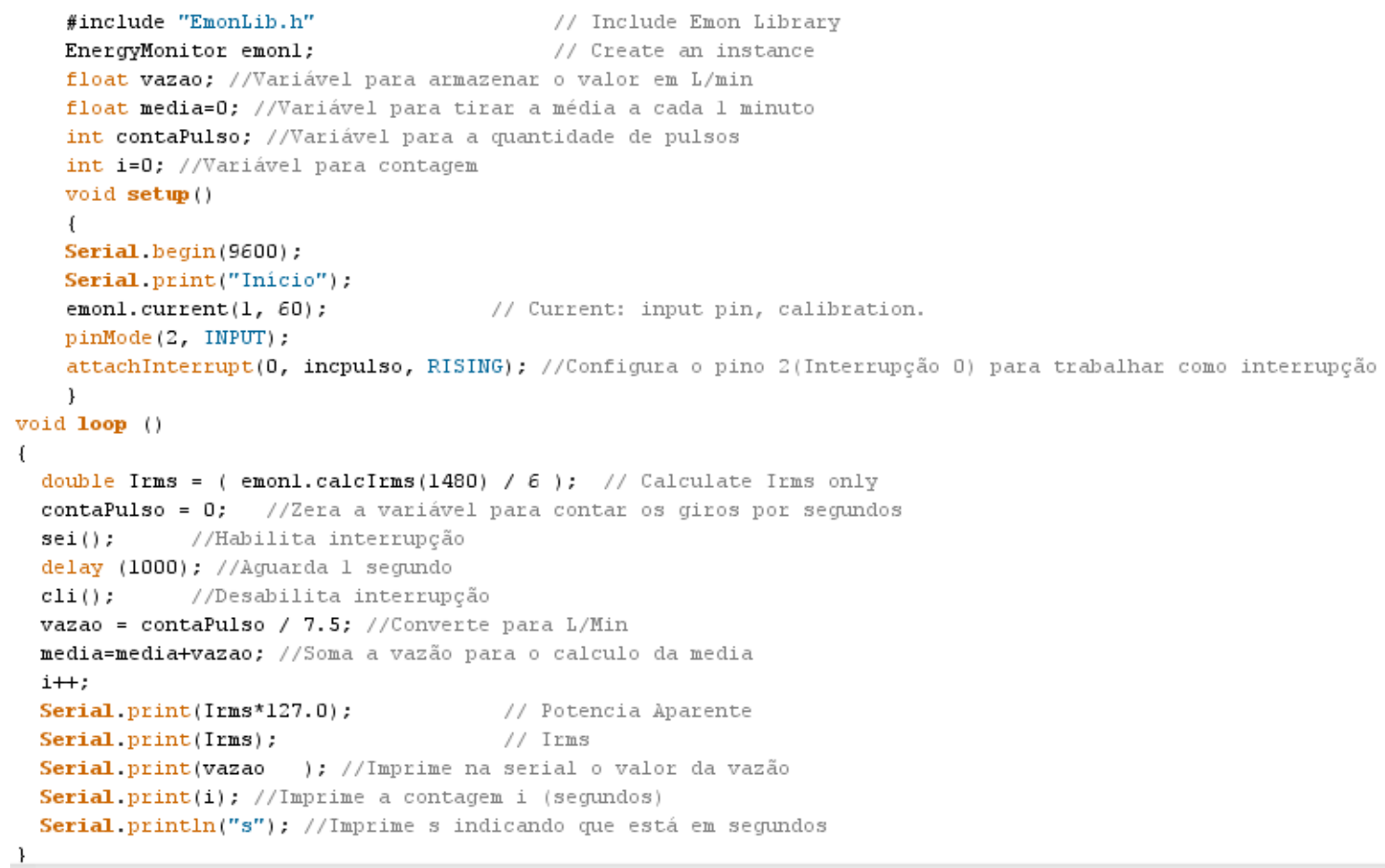

\section{Figura 1 - Código de Programação}

Fonte: Elaborado pelos autores (2015).

Na figura 2, pode ser observada a linha de código onde ocorre a transformação dos pulsos coletados pelo sensor de vazão em litros. É importante ressaltar que o valor 7,5 que está dividindo a variável contaPulso, representa o volume de fluido que passa pelo sensor em um tempo determinado.

vazao = contaPulso/ 7.5;//Converte para L/Min

Figura 2 - Linha de Comando - Calibração do Sensor de Vazão

Fonte: Elaborado pelos autores (2015).

Nota-se na figura 3 o método de calibração utilizado para o cálculo da corrente nominal (IRMS) da instalação. O valor 1480 faz parte das configurações da biblioteca Emolib.h e a divisão por 6 representa o número de voltas dadas pelo fio sobre o sensor.

\section{double Irms $=($ emonl, calcIms $(1480) / 6$ ): // Calculo da Corrente Calibrada}

Figura 3 - Linha de Comando - Calibração do Sensor de Potência

Fonte: Elaborado pelos autores (2015).

\subsection{Hardware}

No decorrer desta seção são mostrados os principais constituintes dos protótipos desenvolvidos. A figura 4 mostra o modelo de placa Arduíno utilizada para acondicionar e tratar os sinais dos sensores de potência e vazão. Foi utilizada a placa Arduíno modelo UNO R3. 


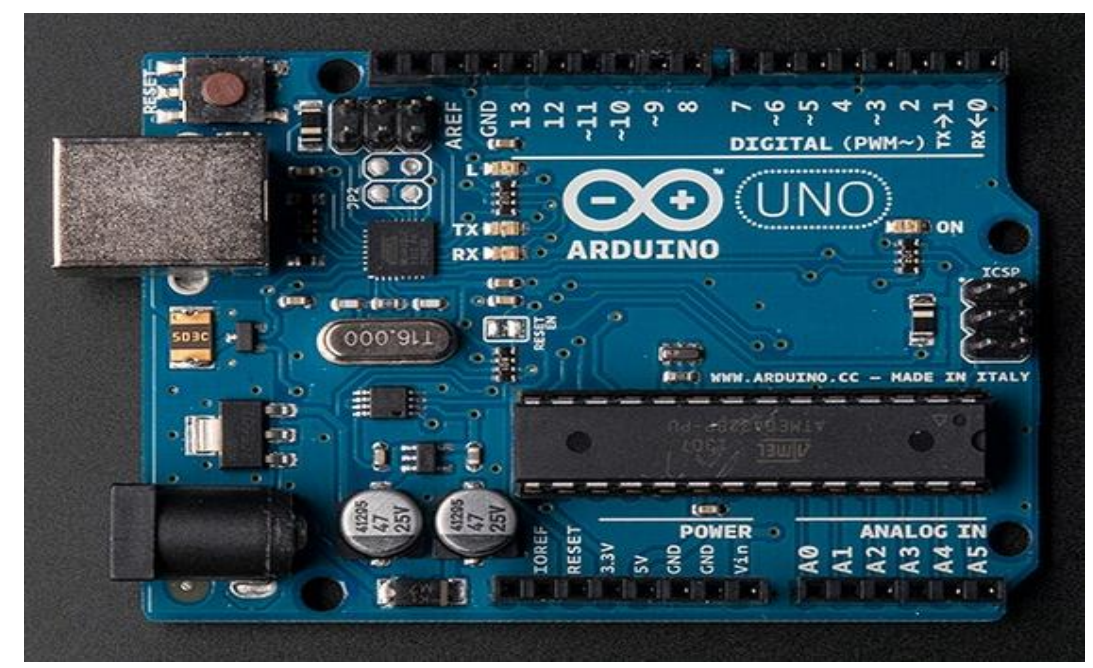

Figura 4 - Arduíno UNO R3

Fonte: Elaborado pelos autores (2015).

A figura 5 mostra o sensor de corrente elétrica modelo SCT-013-000, utilizado como elemento sensor para constituição do sensor de potência. Importante lembrar que pela limitação das entradas analógicas da placa Arduíno, $\pm 5,0 \mathrm{~V}$, foi preciso o uso de um circuito para limitar a saída do sensor a esse intervalo de tensão, conforme mostra a figura 6 .

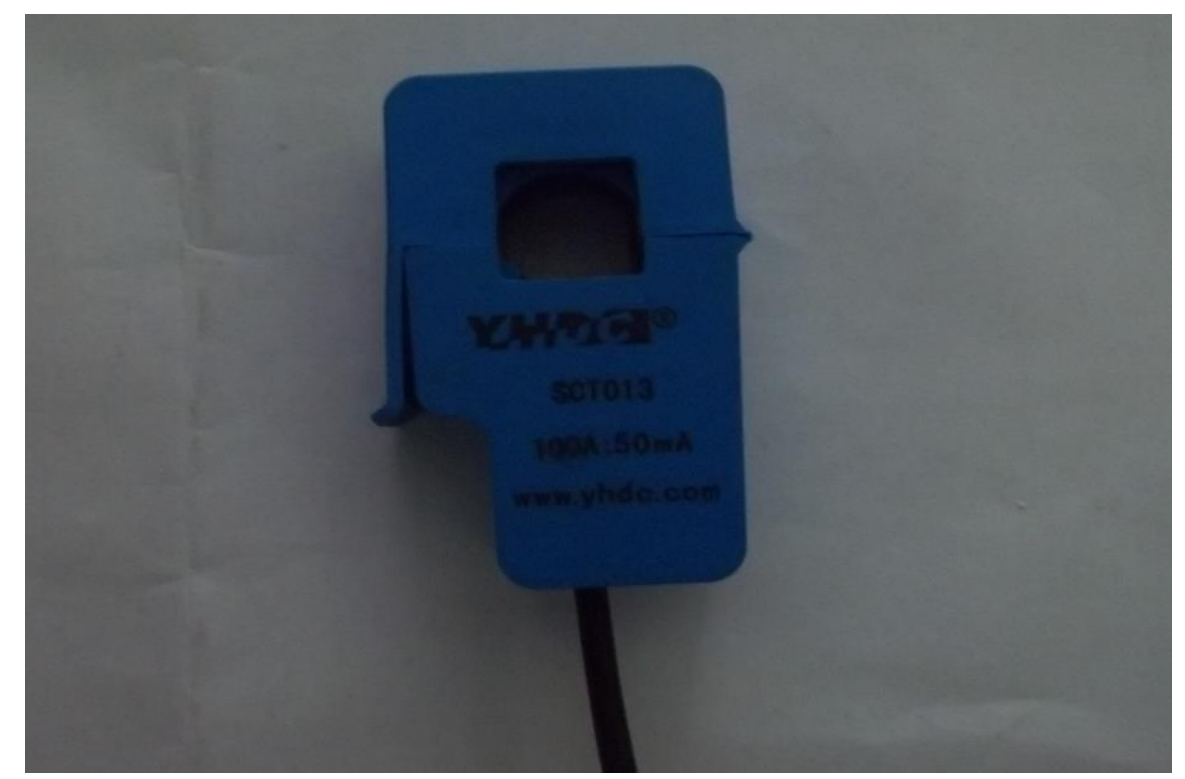

Figura 5 - Sensor de corrente elétrica modelo SCT-013-000

Fonte: Elaborado pelos autores (2015). 


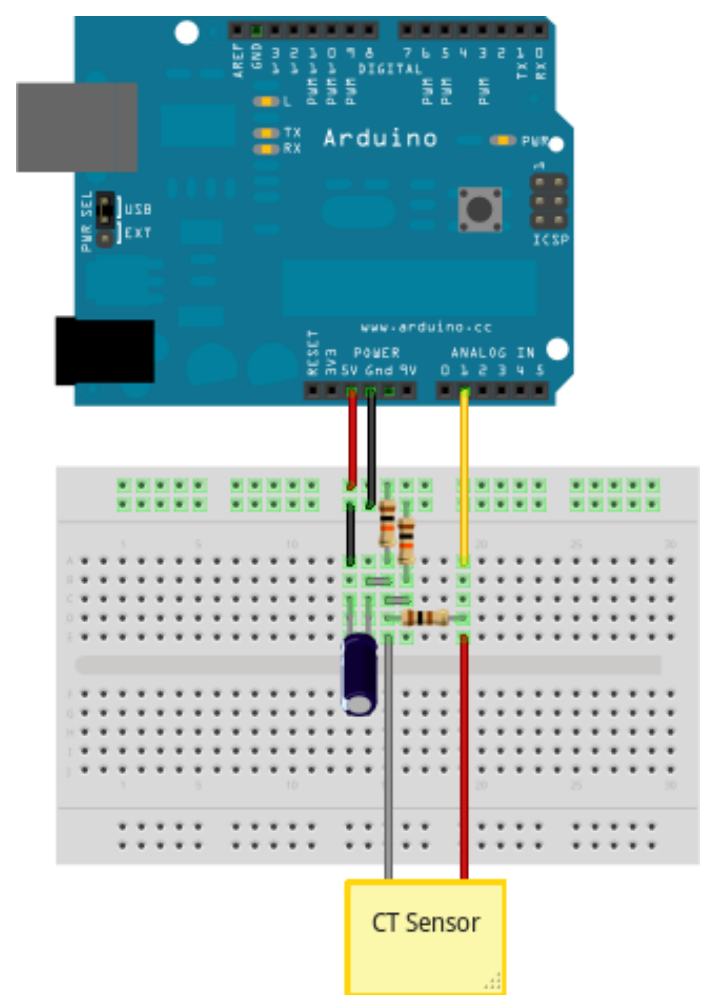

Figura 6 - Circuito Limitador de Tensão do Sensor de Corrente

Fonte: 〈http://openenergymonitor.org/emon/buildingblocks/ct-sensors-introduction>. Acesso em 13 Jul. 2015.

A figura 7 demonstra o sensor de fluxo utilizado para a confecção do sensor de vazão. É possível ver na figura a saída de sinal e de alimentação do sensor (1), assim como a entrada (2) e a saída do sensor (3), ambas com rosca. Na figura 8 é demonstrada a conexão do Arduino com o repectivo sensor.

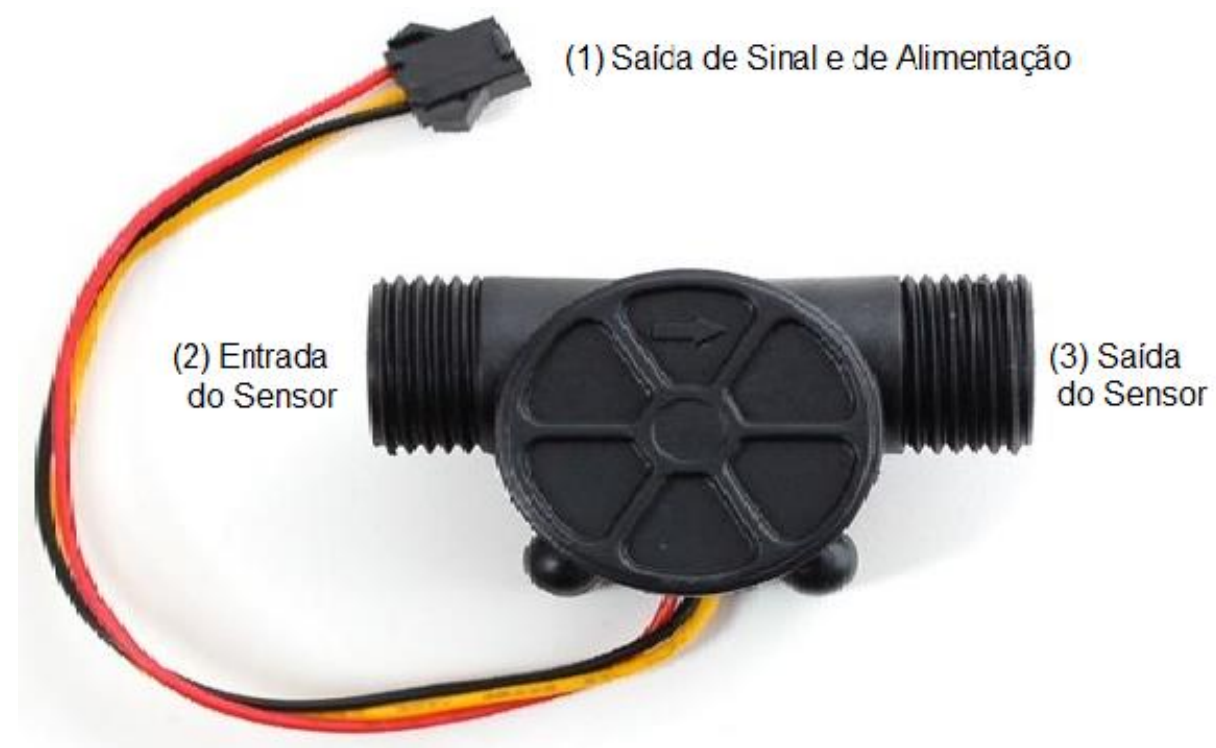

Figura 7 - Sensor fluxo de água ( $1 / 2$ polegada)

Fonte: Elaborado pelos autores (2015). 


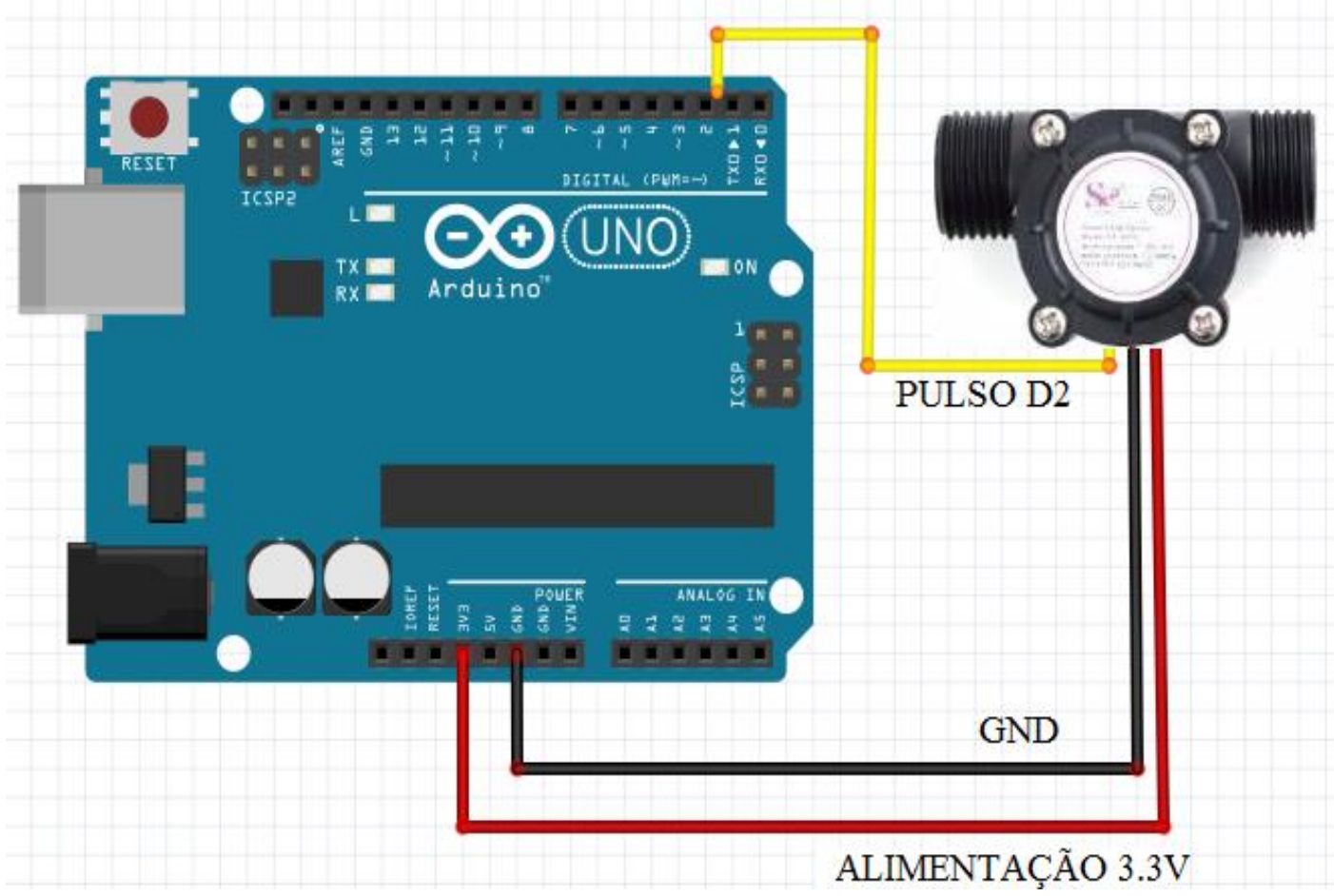

Figura 8 - Ligação do Arduino com o sensor fluxo de água (1/2 polegada) Fonte: Elaborado pelos autores (2015).

As figuras 9 e 10 demonstram os arranjos utilizados para a calibração dos sensores de potência e vazão, respectivamente.

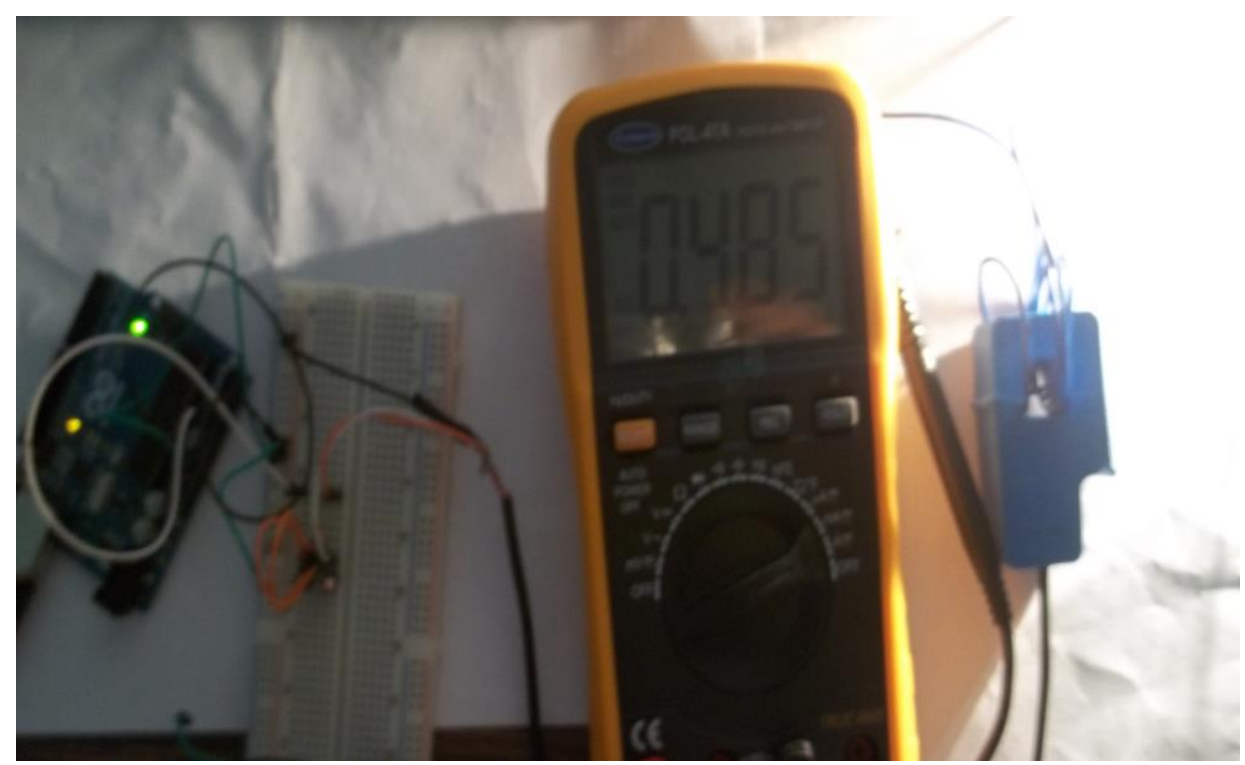

Figura 9 - Arranjo Experimental para calibração do Sensor de Potência Fonte: Elaborado pelos autores (2015). 


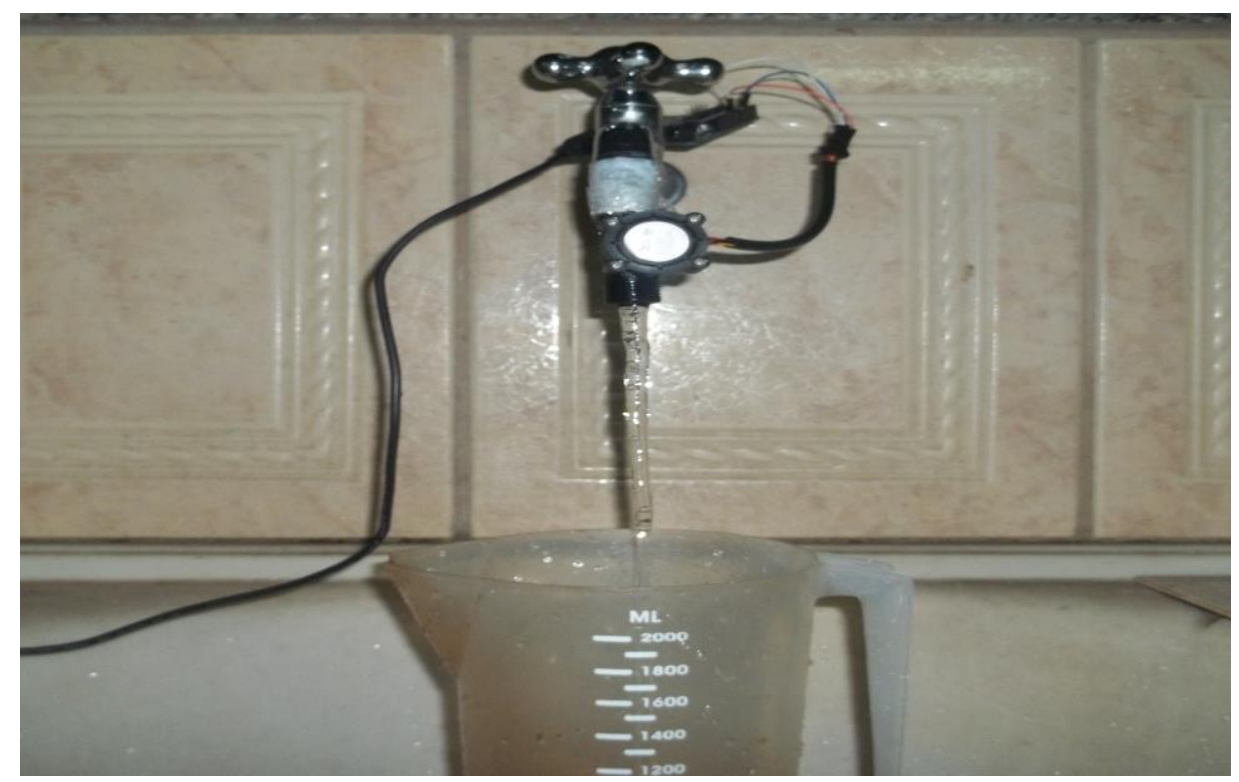

Figura 10 - Arranjo Experimental para calibração do Sensor de Vazão

Fonte: Elaborado pelos autores (2015).

\subsection{Resultados da Calibração}

Após a realização de alguns testes e calibrações descritas anteriormente, foram gerados os gráficos de vazão em função do tempo (Figura 10) e de potência em função do tempo (Figura 11). No caso do teste de vazão, o resultado do método da proveta indicou uma vazão de 0,0366 litros/s, enquanto pelo gráfico podemos ver que o sensor registrou uma vazão média de $0,0333 \mathrm{~L} / \mathrm{s}$, com limite inferior de 0,0298 litros/s e superior de 0,0370 litros/s, caracterizando um erro percentual da ordem de $10 \%$.

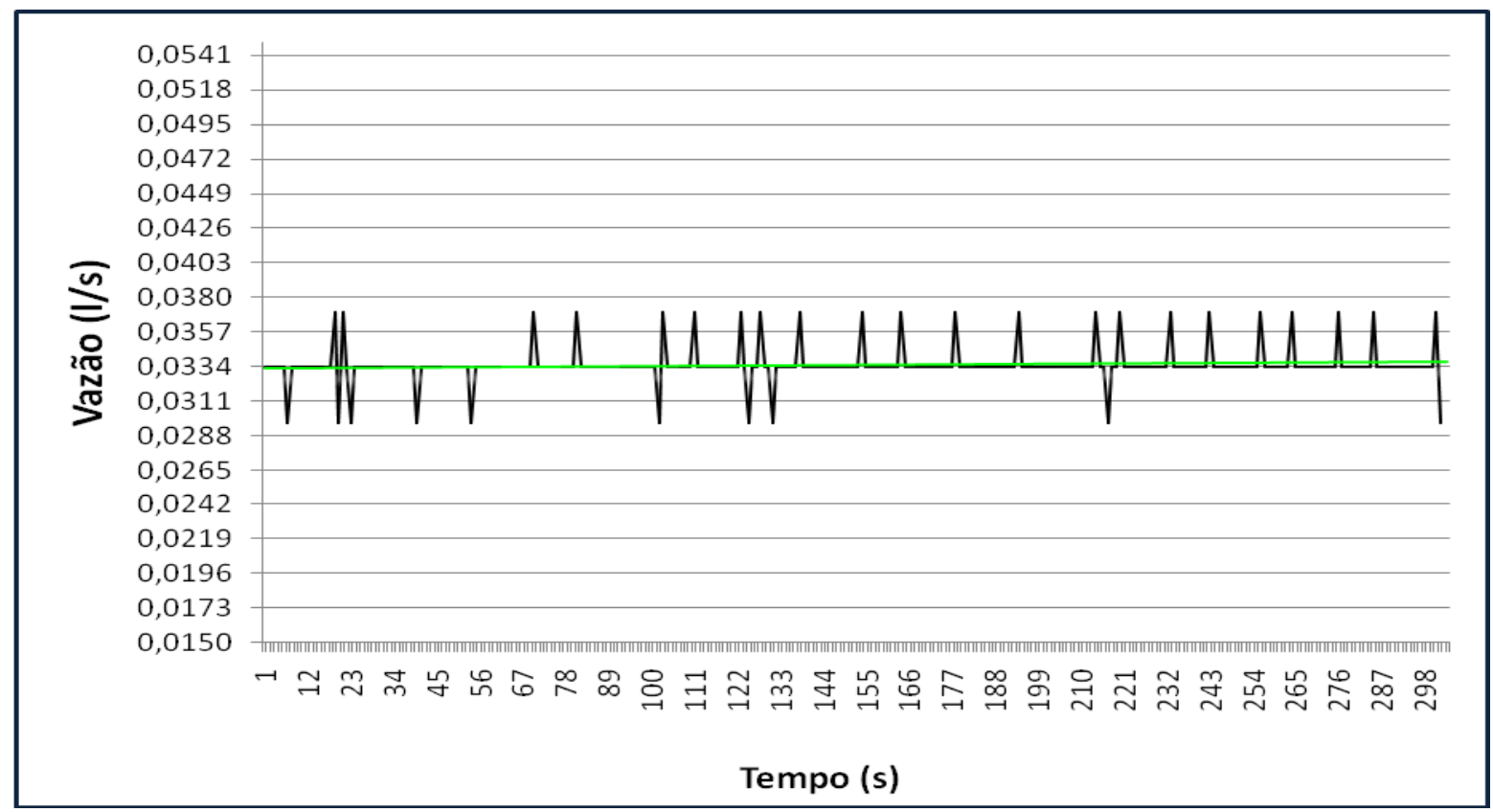

Figura 10 - Gráfico de Vazão como função do tempo Fonte: Elaborado pelos autores (2015). 
O teste do sensor de potência, que foi feito basicamente monitorando a potência de uma lâmpada incandescente de $60 \mathrm{~W}$ de potência nominal, mostrou bastante estabilidade. A potência medida com auxílio do amperímetro indicou potência de $60,0 \mathrm{~W}$, enquanto pelo gráfico podemos ver que o sensor registrou uma potência média de $60,5 \mathrm{~W}$, com limite inferior de $59,7 \mathrm{~W}$ e superior de $61,9 \mathrm{~W}$, caracterizando um erro percentual de $0,83 \%$.

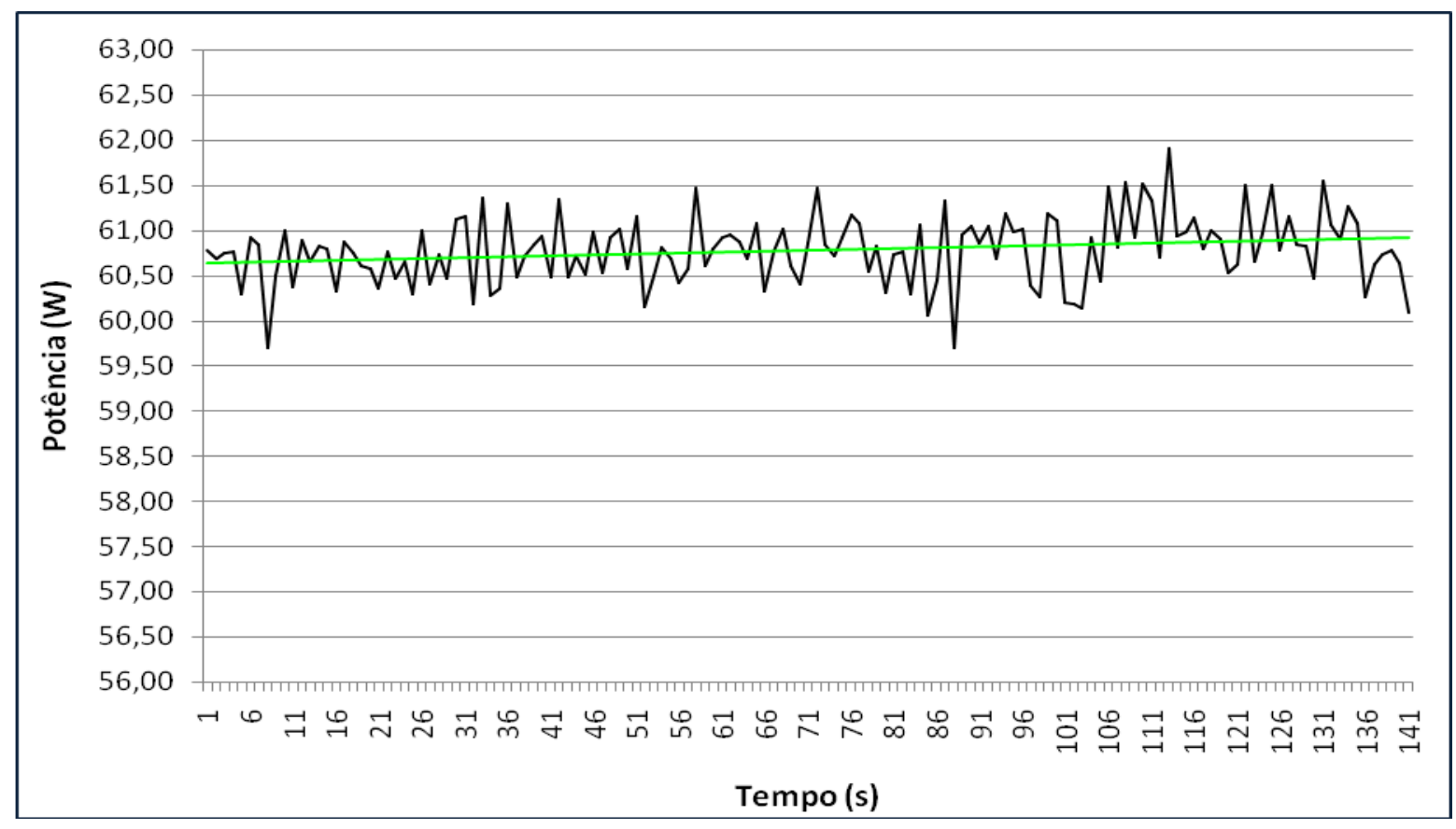

Figura 11 - Gráfico da Potência em função do tempo

Fonte: Elaborado pelos autores (2015).

\section{CONCLUSÃO}

Os resultados dos ensaios de calibração mostraram que os dois sensores produzidos conseguem fazer as medidas com a precisão requerida, dado que o valor do desvio do sensor de potência utilizado no dispositivo foi de $0,83 \%$ e para o sensor de vazão empregado no protótipo obteve um desvio de leitura no valor $9,02 \%$, o que certamente é totalmente aceitável em função do volume de água empregado no mesmo. Haja vista que em proporções maiores de vazão, passa a ser desejável um desvio de leitura menor. Como ainda não se trata de um produto, mas sim de um protótipo pensado para uso como ferramenta de monitoramento e objeto de ensino, tais precisões são compatíveis com a aplicação. No caso da aplicação do sensor de potência, a modelagem baseada na premissa de tensão constante não corresponde a uma barreira técnica significativa, dado que não se quer medir qualidade de energia (afundamentos de tensão, sobretensão, fator de potência, etc). Para o sensor de vazão não há obstáculos, pois o mesmo possui bitola e vazões típicas compatíveis com a rede na qual será instalado.

A próxima etapa será a aplicação dos sensores em um banheiro do IFMG - Câmpus Governador Valadares, com o objetivo de ter as curvas temporais de vazão e potência, obtendo os consumos de água e energia pela simples integração numérica das mesmas. Essas curvas farão parte de um estudo sobre o "padrão" de consumo da referida instalação e poderá auxiliar na tomada de decisão e atividades de conscientização da CICE (Comissão Interna de Conservação de Energia) do campus. Os protótipos também serão usados na disciplina de Recursos Energéticos, como objetos de ensino em atividades experimentais sobre conservação de água e energia. Além disso, está 
prevista a utilização desses equipamentos para compartilhar os conhecimentos acadêmicos adquiridos no desenvolvimento do protótipo com os alunos da instituição em todos os níveis: Graduação, Tecnólogo e Técnico. A ideia é proporcionar possibilidades de ensino em torno do artefato tecnológico desenvolvido, o que proporciona abordagens nas áreas de Eletrônica, Física, Cálculo, Automação Industrial, Estatística, Programação e Gestão Ambiental.

É um passo inicial que permitirá num futuro próximo pensar em desenvolvimento do sistema como produto, maior integração entre as disciplinas e realização de oficinas de aplicação da placa Arduíno para alunos do IFMG - Campus Governador e comunidade externa viam projetos de extensão.

\section{AGRADECIMENTOS}

Agradecemos ao IFMG - Campus Governador Valadares pelo auxílio aos trabalhos, à revista Produção e Desenvolvimento pelo espaço concedido para a publicação e a todos os leitores.

\section{REFERENCIAS}

ANEEL - Agência Nacional de Energia Elétrica. Atlas da Energia Elétrica do Brasil, 3ª ed, Brasília, 2008.

BERMANN, C. Notas sobre la energía incorporada en la exportación de bienes primarios en Brasil. Revista Energia y Equidad, Santa Fe, v. 1, p. 31-38, 2011.

BR-ARDUINO. Arduino e Cartões SD,MicroSD e SDHC. Disponível em: < http://br-arduino.org/2015/01/arduino-ecartoes-sd-microsd-e-sdhc.html>. Acesso em 10 Junho 2015.

DA SILVEIRA, S. A. Inclusão digital, software livre e globalização contra-hegemônica. Software Livre e Inclusao Digital-Organizadores: Sergio Amadeu de Silveira e Joao Cassino, v. 7, p. 11, 2003.

GARCIA, Ricardo Ferreira; DE LIMA, Rodrigo Cavalcante; COIMBRA, Carmen Maria Manhães. AVALIAÇÃO DE UM SENSOR DE FLUXO UTILIZANDO PLACA MICROCONTROLADORA ARDUINO/EVALUATION OF A FLOW SENSOR USING AN ARDUINO MICROCONTROLLER BOARD. Revista Engenharia na Agricultura, v. 23 , n. 2, p. $162,2015$.

KEELLING, RALPH. Gestão de projetos: uma abordagem global. 1a ed. São Paulo: Editora Saraiva, 2012.

MCROBERTS, Michael. Arduino Básico. [tradução. Rafael Zanolli]. 1a ed. São Paulo: Editora Novatec, 2011.

NEWTON, C.Braga. Eletrônica Básica. 5a ed. (2001 - 1a ed.) São Paulo: Editora do Instituto Newton C.Braga, 2012.

OPENNENERGYMONITOR. CT Sensors - $\quad$ An Introduction. Disponível em: <http://openenergymonitor.org/emon/buildingblocks/ct-sensors-introduction>. Acesso em 13 Julho 2015.

PROCOPIO, E.T., et al. Implementação De Medidor De Energia Elétrica Em Plataforma De Hardware Livre Para Estudo Do Comportamento De Redes Inteligentes. XXXIX Congresso Brasileiro de Educação em Engenharia. 2011.

SILVA, L. L. C; MORET, A. S. Impactos Sociais de Hidrelétricas e a Necessidade de Legislação para o Remanejamento. Hydro \& Hydro - CERPCH, v. 54, p. 16-25, 2014.

SOUZA, Anderson R. de et al. The Arduino board: a low cost option for physics experiments assisted by PC. Revista Brasileira de Ensino de Física, v. 33, n. 1, p. 01-05, 2011.

TRATABRASIL, Instituto. Perdas de água dificultam o avanço do saneamento básico e agravam o risco de escassez hídrica no Brasil.Disponível em: <http://www.tratabrasil.org.br/perdas-de-agua-dificultam-o-avanco-do-saneamentobasico-e-agravam-o-risco-de-escassez-hidrica-no-brasil>. Acesso em: 18 Maio 2015.

TIPLER, P.A \& MOSCA, G. Física para Cientistas e Engenheiros. Volume 3. 5a ed. (1998 - $1^{\mathrm{a}}$ ed.) Rio Janeiro: Editora LTC, 2006.

XiDi Tecnology. Datasheet: Splilt-core current transformer. Modelo SCT-013-000. Publicação eletrônica, 2009.

YIFA Ltda. Datasheet: Water Flow Sensor. Modelo YF-21. Publicação eletrônica, 2010. 